\title{
Predicting Intracranial Lesions by Antiplatelet Agents in Subjects with Mild Head Injury.
}

Authors: Andrea Fabbri, M.D., "Franco Servadei, M.D., ${ }^{\S}$ Giulio Marchesini, M.D., "Sherman C Stein M.D., Alberto Vandelli, M.D..

Dipartimento dell'Emergenza, Presidio Ospedaliero Morgagni-Pierantoni, Azienda Unità Sanitaria Locale di Forlì,

*Unità Operativa di Neurochirurgia, Azienda Ospedaliero-Universitaria di Parma, Italy,

${ }^{\S}$ Alma Mater Studiorum, Università di Bologna, Italy.

\#Department of Neurosurgery, University of Pennsylvania School of Medicine, Philadelphia, PA, USA.

Running Head: Antiplatelet therapy and mild head injury

Word Count: 2,848

Correspondence to:

Andrea Fabbri, M.D.

Dipartimento dell'Emergenza

Presidio Ospedaliero Morgagni-Pierantoni

Azienda USL Forlì

Via Forlanini 34, I - 47100 Forlì, Italy (I)

T: +390543735801

F: +390543735880

Email: dr.andrea.fabbri@gmail.com 


\section{SUMMARY}

Background: The effect of pre-injury antiplatelet treatment in the risk of intracranial lesions in subjects after mild head injury (Glasgow Coma Scale 14-15) is uncertain.

Methods: We aimed to determine the potential risk, considering its increasing use in guidelines on cardiovascular disease prevention and aging of the trauma population in Europe.

Patients: We analyzed the interaction of antiplatelet therapy with the prediction variables of main decision aids in 14,288 consecutive adolescent and adult subjects with mild head injury.

Measurements: Any intracranial lesion at CT scan was selected as outcome measure in a multivariable logistic regression analysis.

Findings: Intracranial lesions were demonstrated in 880 cases $(6.2 \%)$, with an unfavorable outcome at 6 months in $86(0.6 \%)$. Antiplatelet drugs were recorded in $10 \%$ of the entire cohort $(24.7 \%$ in the group over 65 years). They increased the risk of intracranial lesions at univaried analysis (odds ratio - OR, 2.6; 95\% confidence interval - 95\% CI 2.2-3.1), interacting with age at multivariate analysis (antiplatelet: OR, 2.7 (1.9-3.7); age $\geq 75$ years, 1.4 (1.0-1.9)). The inclusion of these two variables to those included in previous decision aids for CT scanning (GCS, neurodeficit, posttraumatic seizures, suspected skull fracture, vomiting, loss of consciousness, coagulopathy) predicted intracranial lesions with a sensitivity of 99.7\% (95\% CI 98.9-99.8) and a specificity of $54.0 \%$ (95\% CI 53.1-54.8), with a CT ordering-rate of 49.3\% (undetermined events, 0.2:1000).

Interpretation: Antiplatelet drugs need to be considered in future prediction models on mild head injury, considering their increasing use and progressive aging of the trauma population.

Key words: head injury, intracranial injury, antiplatelet drugs, aging subjects, trauma population. 


\section{INTRODUCTION}

Mild head injury (MHI) with Glasgow coma scale (GCS) 15-14 is low-risk for intracranial complications, but the condition remains a critical issue in terms of disease management because of the potential risk of unfavorable outcome. Nearly $95 \%$ of adult subjects with MHI show no abnormalities on CT scan, and fewer than $1 \%$ need neurosurgical intervention. Every effort should be made to ensure CT scanning for all subjects with intracranial injuries, while limiting the number of negative scans. $\{1\}$

An increasing number of investigations have been carried out to identify subjects at higher risk of intracranial lesions, using groups of predictor variables, in order to increase the specificity of CT scans. $\{2-6\}$ Some of the derived algorhythms have been externally validated $\{6-9\}$ with influence on clinical practice. $\{10-13\}$ A recent study showed that the operating characteristics of the main decision instruments have reached sensitivities over $98 \%$, with specificities ranging from $30 \%$ to $50 \%$, depending on available resources. $\{14\}$

Whereas the use of anticoagulants is a well-known risk factor, the chronic use of antiplatelet drugs has never been considered in the panel of prediction variables tested by the currently available decision aids. This issue was raised in the NICE guidelines, $\{15\}$ but studies of $\underline{\text { hemorrhagic risk attributable to antiplatelet drugs in different groups showed uncertain results. }}\{16$, 17 However, in the last few years the use of antiplatelet drugs has grown considerably. This is due, in part, to national and international guidelines, which promote their widespread use to prevent cardiovascular events in the elderly and other high-risk populations. $\{18,19\}$ This development corresponds to changes in the epidemiology of trauma, which has progressively shifted towards older age-groups, $\{20\}$ in whom antiplatelet drugs use is more prevalent.

Since 1999 a complete database of adolescent and adult subjects with traumatic brain injury has been set up in our hospital, now totaling nearly seventeen thousands of cases. Aim of study was to test the effect of chronic use of antiplatelet drugs on the risk of intracranial lesions in subjects with MHI.

\section{MATERIALS AND METHODS}

\section{Selection of Participants and Interventions}

Consecutive subjects aged 10 years or more who attended the Emergency Department (ED) of the Local Health District of Forlì (I level: 48,000 children and adults per year from a population of 171,000 inhabitants in an area of $1,380 \mathrm{~km}^{2}$ ), within 24 hours of traumatic brain injury. Subjects were enrolled for outcome purposes in a prospective registry, and treated according to the recommendations of the Neurotraumatology Committee of the World Federation of Neurosurgical Societies. $\{14\}$ 
Fabbri $/ 4$

\section{Database}

The Head Injury Registry consists of 16,799 consecutive medical records of all subjects attending within 24 hours from the event (from Jan 99 to Dec 07). MHI was defined as an acute injury of the head, other than any superficial injury to the face, GCS 14 or 15 in adolescent and/or adult inhabitants aged $\geq 10$ years. 2,511 records (14.9\%) were excluded because: a) unclear history of injury as the primary event (1,100 cases); b) unstable vital signs (378 cases); c) GCS <14 (302 cases); d) penetrating injuries at presentation (33 cases); e) discharge against medical advice (388 cases), f) duplicate records of subjects re-attending for post-concussive syndrome within 7 days of admission for MHI (310 cases).

The use of aspirin as antiplatelet drug (any dosage) was systematically recorded, independent of time of exposure. Indobufen (a second antiplatelet drug used in Italy) was also considered. On the contrary, we did not consider the potentially antiplatelet activity of other anti-inflammatory agents. Finally, also clopidogrel was not included in the analysis because it was not on the market at time of protocol set-up, and was later very rarely used in this old population, also due to regulatory limitations in Italy.

\section{$\underline{\text { Treatment Protocol }}$}

Subjects were treated according to a predefined diagnostic and observational protocol as previously published. $\{21\}$ Briefly, low-risk patients were sent home after examination without any radiological imaging. Medium-risk patients were submitted to CT scan according to physicians' judgement, and discharged after at least 3-24 hours from trauma in the case of negative results. High-risk patients were submitted to CT imaging and managed with a first 24 to 48-hour strict observation period, regardless of CT findings. Age over 60 was not considered per se a risk factor.

All subjects re-attending the ED were submitted to CT scans.

CT scans showing intracranial lesions were reviewed urgently by a neurosurgeon of the nearest Neurosurgical Unit (Cesena, $17 \mathrm{~km}$ away), consulted via a teleradiology system. The protocol was carried out according to the Helsinki Declaration and approved by the ethical committee of the Local Health District of Forlì.

\section{Variable Definition and Outcome Measures}

The analysis was based on the pool of variables considered in main decision instruments of the literature $\{14\}$ and the chronic use of antiplatelet therapy as additional variable (Table 1).

The main outcome measure was the diagnosis of any post-traumatic lesion at CT within 7 days from trauma. Lesions included: a) intracerebral hematomas/brain contusion(s) (ICH); b) traumatic subarachnoid hemorrhage (t-SAH); c) subdural hematoma (SDH); d) epidural hematoma (EDH), e) depressed skull fracture (DSF). $\{3\}$ 
In subjects revisited for complaints a time limit of 7 days was set to consider any lesion as directly related to the initial head injury. Similarly, hematoma evacuation and/or skull fracture elevation were only considered within 7 days after diagnosis. Late events were not considered in the present analysis.

The 6-month outcome was carried out by a systematic search of all patients was carried out by checking the death certificates and the medical databases of our local health district. Patients without post-traumatic lesions and not readmitted for complaints were considered fully recovered. In addition, a member of the ED staff contacted all patients with a defined post-traumatic lesion and rated GOS by means of a structured telephone interview. $\{22\}$ The outcome was considered unfavorable if the patient died for head injury-related causes, remained in a vegetative state, or had a permanent severe disability.

\section{Analyses}

Mean value, standard deviation (SD), median, inter-quartile range (IQR) and frequencies were used to describe data distribution. We used multivariable logistic regression with forward stepwise selection with a $P$ value greater than 0.05 for removal of variables. The odds ratio $(\mathrm{OR})$ and $95 \%$ confidence intervals (95\% CI) were also calculated. Sensitivity and specificity were evaluated, together with the negative predictive value (NPV). We tested associations of each risk factor with the primary outcome measure using chi-square tests for nominal variables, the Mann-Whitney U test for ordinal variables, and the unpaired 2-tailed $t$-test for continuous variables by using SPSS software, version 17.0 (SPSS Inc., Chicago, Illinois). We also assessed the operating characteristics by calculating the area under the receiver operating characteristic (ROC) curve for variables selected by the multivariable logistic regression analysis. Proportions were compared between protocols by means of Fisher's exact test.

\section{RESULTS}

\section{Patient population}

The main predicting variables associated with intracranial injuries are reported in Table 2.

The median age of the 14,228 subjects was 49 years (IQR, 29 - 75), with 5,180 (36.4\%) $\geq 65$ years and as many as $3,701(26.0 \%) \geq 80$. Most subjects were injured following a road accident $5,990(42.1 \%)$ and falls or accidental reasons (5,379 cases, 37.8\%). In the remaining subjects the head injury was work-related in 1,022 (7.2\%), assault in 503 (3.5\%), and related to sport and other causes in $732(5.1 \%)$. Adherence to the local protocol was nearly complete, with a CT scan ordering-rate of $9,056(63.6 \%)$ of total cases. 
Antiplatelet drug use (excluding clopidogrel) was recorded in $10 \%$ of the entire cohort $(24.7 \%$ in the group $\geq 65$ years), and was associated with the risk of intracranial lesions (OR, 2.6; 95\%CI, 2.2 - 3.1: P <0.001) (Table 2).

A total of 1,201 injuries were detected in 880 subjects (6.1\%), with a single lesion in 600 (4.0\% respectively). Lesions included 591 intraparechymal hemorrhagic contusions, 329 acute subdural, 63 acute epidural hematomas, 193 subarachnoid hemorrhages and 25 depressed skull fractures.

Neurosurgical intervention was performed in 153/880 (17.3\%) subjects within 7 days for acute subdural hematomas (66 cases), depressed skull fractures (17 cases), epidural hematomas (26 cases), intracerebral hematomas/brain contusion (96 cases) in different combinations.

Follow-up data were obtained in 715 cases with intracranial lesions $(87.0 \%)$. Of the remaining 107 cases, 16 subjects died because of head injury-unrelated complications, 18 were lost to followup, and in 73 GOS was unreliable, due to either a previous disability (68 cases) or a disability unrelated to head injury (5 cases). The outcome was unfavorable in 86 cases $(0.6 \%)$, with 51 aged $\geq 75$ years. 36 subjects died, 2 remained in a permanent vegetative state $(0.1 \%)$ and 62 cases $(0.4 \%)$ were severely disabled. Only 76 cases had a moderate disability and 600 fully recovered.

\section{Outcome Prediction}

Eight of the 14 items proposed were independently associated with intracranial lesions (Table 3), but only 4 (GCS, suspected skull fracture, neurodeficit and loss of consciousness) showed a good discriminant capacity ( $\mathrm{ROC}$ areas $>0.65$ ) in predicting an intracranial lesion, the remaining having little or no discriminant ability.

The discriminating operating characteristics area of the items selected was 0.697 (95\% CI 0.690 - 0.705), sensitivity 98.4 (95\% CI 97.3 - 99.0), specificity 41.1 (95\% CI 40.2 - 41.9) with a theoretical CT ordering rate of $61.4 \%$ and only 14 subjects, with intracranial lesions remaining undetermined (EDH, 2 cases; ICH, 4; SDH, 7; SDH + ICH, 1) (Table 4).

When the covariate pre-injury use of antiplatelet drugs was added into the model, it showed a significant additional effect (OR 2.8 (95\% CI 2.0 - 3.9: $P<0.001$ ]. Antiplatelet therapy entered the model as predictor variable excluding age $\geq 65$ years $(\mathrm{OR} 1.2,95 \% \mathrm{CI} 0.9-1.7 ; \mathrm{P}=0.202)$ (Table 4). The discriminating operating characteristics area of the new item combination was higher in comparison to that of the classical items $(0.807,95 \%$ CI $0.803-0.814$; Fisher exact test; $\mathrm{P}<0.001)$ (Table 4). This increased specificity by $18.6 \%$, lowered the CT ordering rate, but 6 more cases remained undetermined compared with the classical model (ICH, 3 cases; SDH, 3 cases) (Table 4). All these cases were $\geq 80$ years old, had non-neurosurgical lesions and had a good recovery with the exception of 1 patient, who died at 6 months.

The interrelationship of age with antiplatelet use was further tested at different age cut-offs 
(Appendix Table 1). Finally, a model was built, including both age $\geq 75$ years and antiplatelet therapy, that showed a significantly lower discriminating operating characteristics area 0.783 (95\%CI $0.761-0.775 ; \mathrm{P}<0.001)$, a 9.8\% decrease in specificity, without significant differences in sensitivity (Table 4). The CT ordering rate was now around 50\%, and only 3 cases remained undiagnosed (EDH, 2 cases, 20 yrs and 59 yrs, respectively; ICH, 1 case, 63 yrs). Of these only the 59 yrs old man with EDH was operated with unfavorable outcome. The remaining 2 subjects had a good recovery at six months. The 3 cases had no symptoms or risk factors, and their lesions would remain undetected by any clinical decision instrument.

\section{DISCUSSION}

This observational analysis shows that pre-injury antiplatelet therapy significantly increases the risk of intracranial lesions in subjects after MHI, and should be considered in future reviews of the predictive algorhythms on this topic. The inclusion of antiplatelet drugs in our prediction model shows a good accuracy and reduces to a minimum the number of undiagnosed lesions $(<0.2 / 1000)$ with a CT ordering rate of $49 \%$. Also the type of items (age $\geq 75$ years and chronic antiplatelet therapy) may be more reliable than the association of age $\geq 65$ in the presence of amnesia or loss of consciousness. $\{2\}$

Antiplatelet therapy, mainly aspirin, is commonly prescribed to reduce cardiovascular morbidity and mortality in subjects with cardiovascular disease over 45 years. $\{18\}$ Its use has increased considerably in the general Italian population in the last few years, namely in high-risk subjects. A recent analysis of drug prescribing in over 300.000 Italian subjects with diabetes showed an increased use of antiplatelet drugs from $15 \%$ to $52 \%$ in the period 1997-2006, $\{23\}$ and in 2008 over $4 \%$ of the Italian population was treated with aspirin. $\{24\}$ The outcome was determined 6 months after injury, by checking the death certificates and the hospital database and by a structured telephone interview, to determine the presence of disability.

In our database, nearly $10 \%$ of the whole population was treated with antiplatelet agents, and increased to $24.7 \%$ in the group of subjects over 65 years. This problem is expected to become more and more relevant, following guidelines, indicating the need for antiplatelet drug therapy in a large proportion of older subject. $\{18\}$ Although the effects on cardiovascular thrombosis is definitely proved, antiplatelet drugs were reported to increase the occurrence of chronic subdural hemorrhage in the elderly, $\{25\} '\{26\}$ as well as morbidity and mortality in older subjects with intracranial post traumatic hemorrhage. $\{27\},\{28\}$

There is a small body of literature on intracranial hemorrhage and aspirin use outside spontaneous events. In a small sample with mild-to-moderate head injury, the mortality rate of subjects receiving aspirin was higher-than-normal, $\{16\}$ but in a prospective study low-dose aspirin 
prophylaxis showed no effect in the frequency or types of intracerebral or meningeal hemorrhage in subjects over 60 years following mild and moderate head injury. $\{17\}$

According to these considerations and the availability of a large database, we looked at chronic antiplatelet drug treatment as a risk factor, potentially adding to the 14 variables considered by the main clinical decision strategies. $\{5,14\}$ In the multivariable analysis the use of antiplatelet therapy improved the prediction of the covariate panel in the logistic model, and increased the age cut-off to 75 years in a sensitivity analysis (Table 4). The age limit of 75 years was empirically selected on the basis of repeated analyses testing age at 5-year intervals. Data were not corrected for multiple testing and should be confirmed in different settings. The decision about which age threshold should be used (that is, the minimum desired sensitivity) should ideally be based on a cost/benefit analysis of CT scanning. $\{14\}$ In the clinical setting, there is evidence that prediction rules are more and more frequently used by busy physicians as decision aids, i.e., they use predicted probabilities of an outcome in the decision-making process. $\{14\}$ The inclusion of antiplatelet therapy and age over 75 as new cut-off, irrespective of clinical symptoms, to main accepted variables provides the best sensitivity (99.7\%), a good level of specificity (54\%), an acceptable CT ordering rate (49.3\%), thus reducing to a minimum the number of undetermined lesions (Table 4).

In a few models, age is considered a prediction variable only in the presence of amnesia or loss of consciousness. $\{2,3,29\}$ However, the accepted age cut-offs of the main guidelines (60 or 65 years) are associated with a very low specificity and an unacceptably high CT ordering rate. In our protocol, old age, independently of signs and symptoms was not considered an a priori risk factor, since it would increase CT ordering rate over $70 \%$ without improving the number of undetermined lesions. $\{7\}$ Also limiting the use of age to subjects with loss of consciousness or amnesia gives a CT ordering rate over $60 \%$. Old subjects are more likely to develop a head injury by a fall mechanism, $\{29\}$ have mortality rates two-fold higher than younger subjects, $\{30\}$ a higher number of complications and need of extended care after discharge. $\{31\}$ However, not age per se, but a series of conditions associated with age, probably accounts for the increased risk, and the concurrent use of antiplatelet therapy might be pivotal.

Limitations: Firstly, the clinical characteristics of subjects were obtained from variables registered on a prospective database, but all analyses were retrospectively performed, potentially creating a bias to results. Secondly, the results are based on a single-centre large database, with only one district reference hospital, with a possible selection bias. An external validation in different settings is needed. Thirdly, elderly subjects may be unable to recollect their medications, and a few cases might have been missed. Given that not all subjects had a CT scan and the assumption that no lesions were present in subjects who did not have a scan and were not readmitted later, the above 
sensitivity refers to clinically significant lesions. This is likely to underestimate the true sensitivity of predictor variables for intracranial lesions, but missing clinically silent lesions is acceptable to reduce costs, provided that a rescue mechanism is operative. Our ED is the core of a community hospital, serving a well-defined population, and we previously reported that possible missing cases did not have an unfavorable outcome, as assessed by death certificates $\{32\}$.

In conclusion, antiplatelet drug therapy is a significant risk factor for the development of intracranial lesions after MHI. The use of these drugs issue has increased in the aging population, which also explain the lack of significance reported in previous analyses, and should be considered in future guidelines revisions. In our hands, antiplatelet drug use, coupled with age $\geq 75$ years, independently on amnesia and loss of consciousness, when included as risk factors together with the classical, main prediction variables, reduce to a minimum (0.2:1000) the number of cases with undetermined intracranial lesions, with a CT ordering rate of $49 \%$. These results need external validation in different settings to be proposed in a new decision aid.

\section{CONTRIBUTORS}

AF conceived the study, wrote the protocol, coordinated the data collection, interpretation of results, and wrote the paper. FS contributed to interpretation of the results and critical review of the paper. GM, contributed to study design, interpretation of the results, and co-wrote the paper. SS, contributed to interpretation of the results and critical review of the paper and co-wrote the paper. AV contributed to study design, study coordination, interpretation of the results, and critical review of the paper. All authors approved the final version of the paper.

\section{CONFLICT OF INTEREST.}

All Authors warrant to have no conflict of interest in connection with this paper, they have access to all data in the study and they held final responsibility for the decision to submit for publication

\section{ACKNOWLEDGMENTS.}

We are grateful to the Emergency Medical System personnel of the Azienda USL of Forlì for helpful support.

\section{FUNDING •}

None.

\section{LiCENCE for Publication:}

The Corresponding Author has the right to grant on behalf of all authors and does grant on behalf of all authors, an exclusive licence (or non exclusive for government employees) on a worldwide basis to the BMJ Publishing Group Ltd to permit this article (if accepted) to be published in PMJ and any other BMJPGL products and sublicences such use and exploit all subsidiary rights, as set out in our licence. 


\section{REFERENCES:}

1. Jagoda AS, Bazarian JJ, Bruns JJ, Jr., et al.: Clinical policy: neuroimaging and decisionmaking in adult mild traumatic brain injury in the acute setting. Ann Emerg Med 2008; 52: 714-748

2. Haydel MJ, Preston CA, Mills TJ, et al.: Indications for computed tomography in patients with minor head injury. N Engl J Med 2000; 343: 100-105

3. Stiell IG, Wells GA, Vandemheen K, et al.: The Canadian CT Head Rule for patients with minor head injury. Lancet 2001; 357: 1391-1396

4. Servadei F, Teasdale G, Merry G: Defining acute mild head injury in adults: a proposal based on prognostic factors, diagnosis, and management. J Neurotrauma 2001; 18: 657-664

5. Smits M, Dippel DW, Steyerberg EW, et al.: Predicting intracranial traumatic findings on computed tomography in patients with minor head injury: the CHIP prediction rule. Ann Intern Med 2007; 146: 397-405

6. Dunning J, Lecky F: The NICE guidelines in the real world: a practical perspective. Emerg Med J 2004; 21: 404-407

7. Fabbri A, Servadei F, Marchesini G, et al.: Clinical performance of NICE recommendations versus NCWFNS proposal in patients with mild head injury. J Neurotrauma 2005; 22: 14191427

8. Smits M, Dippel DW, de Haan GG, et al.: External validation of the Canadian CT Head Rule and the New Orleans Criteria for CT scanning in patients with minor head injury. Jama 2005; 294: 1519-1525

9. Stiell IG, Lesiuk H, Wells GA, et al.: Canadian CT head rule study for patients with minor head injury: methodology for phase II (validation and economic analysis). Ann Emerg Med 2001; 38: 317-322

10. Bramley R, Whitehouse RW, Taylor PM: The Canadian CT Head Rule for patients with minor head injury: consequences for radiology departments in the U.K. Clin Radiol 2002; 57: 151-152; author reply 152-153

11. Muller K, Waterloo K, Romner B, et al.: Mild head injuries: impact of a national strategy for implementation of management guidelines. J Trauma 2003; 55: 1029-1034

12. Shravat BP, Huseyin TS, Hynes KA: NICE guideline for the management of head injury: an audit demonstrating its impact on a district general hospital, with a cost analysis for England and Wales. Emerg Med J 2006; 23: 109-113

13. Sultan HY, Boyle A, Pereira M, et al.: Application of the Canadian CT head rules in managing minor head injuries in a UK emergency department: implications for the implementation of the NICE guidelines. Emerg Med J 2004; 21: 420-425

14. Stein SC, Fabbri A, Servadei F, et al.: A critical comparison of clinical decision instruments for computed tomographic scanning in mild closed traumatic brain injury in adolescents and adults. Ann Emerg Med 2009; 53: 180-188

15. National Institute for Clinical Excellence. Head Injury Triage, Assessment, Investigation and Early Management of Head Injury in Infants, Children and Adults. Clinical Guideline 56, 2007; London, England: National Collaborating Centre for Acute Care at the Royal College of Surgeons of England, available at: http://www.nice.org.uk/CG56

16. Spektor S, Agus S, Merkin V, et al.: Low-dose aspirin prophylaxis and risk of intracranial hemorrhage in patients older than 60 years of age with mild or moderate head injury: a prospective study. J Neurosurg 2003; 99: 661-665

17. Ivascu FA, Howells GA, Junn FS, et al.: Predictors of mortality in trauma patients with intracranial hemorrhage on preinjury aspirin or clopidogrel. J Trauma 2008; 65: 785-788

18. Smith SC, Jr., Blair SN, Bonow RO, et al.: AHA/ACC Scientific Statement: AHA/ACC guidelines for preventing heart attack and death in patients with atherosclerotic cardiovascular disease: 2001 update: A statement for healthcare professionals from the 
American Heart Association and the American College of Cardiology. Circulation 2001; 104: $1577-1579$

19. Patrono C, Bachmann F, Baigent C, et al.: Expert consensus document on the use of antiplatelet agents. The task force on the use of antiplatelet agents in patients with atherosclerotic cardiovascular disease of the European society of cardiology. Eur Heart $\mathbf{J}$ 2004; 25: 166-181

20. Giannoudis PV, Harwood PJ, Court-Brown C, et al.: Severe and multiple trauma in older patients: incidence and mortality. Journal of Trauma 2009; [Epub February 11, 2009, ahead of print]

21. Fabbri A, Servadei F, Marchesini G, et al.: Prospective validation of a proposal for diagnosis and management of patients attending the emergency department for mild head injury. J Neurol Neurosurg Psychiatry 2004; 75: 410-416

22. Thornhill S, Teasdale GM, Murray GD, et al.: Disability in young people and adults one year after head injury: prospective cohort study. BMJ 2000; 320: 1631-1635

23. De Rosa M, Marchesini G: Osservatorio ARNO diabete. Analisi di 10 anni di prescrizioni, in Collana Rapporti ARNO. Bologna, CINECA, Consorzio interuniversitario, 2007, pp. 147

24. Osservatorio Nazionale sull'impiego dei medicinali: L'uso dei Farmaci in Italia. Roma, ISSAIFA, 2008

25. Reymond MA, Marbet G, Radu EW, et al.: Aspirin as a risk factor for hemorrhage in patients with head injuries. Neurosurg Rev 1992; 15: 21-25

26. Stroobandt G, Fransen P, Thauvoy C, et al.: Pathogenetic factors in chronic subdural haematoma and causes of recurrence after drainage. Acta Neurochir (Wien) 1995; 137: 6-14

27. Ohm C, Mina A, Howells G, et al.: Effects of antiplatelet agents on outcomes for elderly patients with traumatic intracranial hemorrhage. J Trauma 2005; 58: 518-522

28. Mina AA, Knipfer JF, Park DY, et al.: Intracranial complications of preinjury anticoagulation in trauma patients with head injury. J Trauma 2002; 53: 668-672

29. Fortuna GR, Mueller EW, James LE, et al.: The impact of preinjury antiplatelet and anticoagulant pharmacotherapy on outcomes in elderly patients with hemorrhagic brain injury. Surgery 2008; 144: 598-603; discussion 603-595

30. Mosenthal AC, Livingston DH, Lavery RF, et al.: The effect of age on functional outcome in mild traumatic brain injury: 6-month report of a prospective multicenter trial. $\mathrm{J}$ Trauma 2004; 56: 1042-1048

31. Susman M, DiRusso SM, Sullivan T, et al.: Traumatic brain injury in the elderly: increased mortality and worse functional outcome at discharge despite lower injury severity. J Trauma 2002; 53: 219-223; discussion 223-214

32. Fabbri A, Servadei F, Marchesini G, et al.: Observational approach to subjects with mild-tomoderate head injury and initial non-neurosurgical lesions. J Neurol Neurosurg Psychiatry 2008; 79: 1180-1185 


\section{Table 1}

The main clinical characteristics associated with a high risk of clinically significant outcomes in subjects with mild head injury

\section{Variable}

GCS

Amnesia

Suspected skull fracture

Vomiting

Age

Coagulopathy

Neurological deficit

Seizure

Loss of consciousness

Visible trauma

Headache

Injury mechanism

Intoxication

Previous neurosurgical intervention

Antiplatelet Drugs

\section{Definition}

Glasgow coma scale 15 or 14 after stabilization in the ED,

A short-term memory deficit or amnesia, and deemed amnesia present for the traumatic event if the patient could not recall the entire traumatic event,

Clinical signs of fracture; other injuries, such as contusions, lacerations, or abrasions were classified as visible trauma,

More than one episode of vomiting in adults,

Subjects $\geq 65$ years, provided they had experienced some loss of consciousness or amnesia,

History of bleeding, clotting disorder, current treatment with warfarin. Non-iatrogenic coagulopathy, considered a risk factor, was simply assessed by patient history.

Abnormality on routine clinical neurologic examination that indicated a focal cerebral lesion.

A witnessed or suspected seizure after the injury, without an history of epilepsy.

A loss of consciousness reported by a witness or by the patient itself.

A clinically significant discontinuity of the skin or injury above the clavicles.

Headache only as being diffuse.

Dangerous mechanisms of injury (a pedestrian or cyclist struck by a motor vehicle, an occupant ejected from a motor vehicle or a fall from a height of greater than $1 \mathrm{~m}$ or five stairs).

Blood alcohol levels $>100 \mathrm{mg} / \mathrm{dL}$ or positive urine tests for drugs of abuse requested by the physician on the basis of clinical suspicion.

History of neurosurgical interventions.

Platelet aggregation inhibitors treatment: pre-injury therapy with Aspirin $100 \mathrm{mg}$, Ticlopidine $250 \mathrm{mg}$, Indobufen 200 mg. Subjects taking Clopidogrel were excluded by the present analyses. 


\section{Table 2}

Risk of intracranial lesions after mild head injury in relation to the presence of the main predicting variables identified by previous decision instruments.

\begin{tabular}{lcccc}
\hline & With Lesions & Without Lesions & OR (95\% CI) & P value \\
Variables & $(\mathbf{N}=\mathbf{8 8 0})$ & $\mathbf{( N = 1 3 , 3 4 8 )}$ & & \\
GCS 14 & & & & \\
Amnesia & $565(64.2 \%)$ & $253(1.9 \%)$ & $92.8(77.1-111.8)$ & $<0.001$ \\
Suspected skull fracture & $286(32.5 \%)$ & $86(0.6 \%)$ & $74.2(57.6-95.8)$ & $<0.001$ \\
Vomiting & $173(19.7 \%)$ & $204(1.5 \%)$ & $15.8(12.7-19.6)$ & $<0.001$ \\
Age $\geq 65$ yrs* & $430(48.9 \%)$ & $2,947(22.1 \%)$ & $3.4(2.9-3.9)$ & $<0.001$ \\
Age $\geq 75$ yrs & $352(40.0 \%)$ & $3,349(25.1 \%)$ & $2.0 .(1.7-2.3)$ & $<0.001$ \\
Coagulopathy & $123(14.0 \%)$ & $302(2.3 \%)$ & $7.0(5.6-8.8)$ & $<0.001$ \\
Neurological deficit & $485(55.1 \%)$ & $49(0.4 \%)$ & $333.0(244.3-454.5)$ & $<0.001$ \\
Seizure & $77(8.8 \%)$ & $185(1.4 \%)$ & $6.8(5.2-9.0)$ & $<0.001$ \\
LOC & $629(71.5 \%)$ & $3,601(27.0 \%)$ & $6.8(5.8-7.9)$ & $<0.001$ \\
Visible trauma & $690(78.4 \%)$ & $9,129(68.4 \%)$ & $1.7(1.4-2.0)$ & $<0.001$ \\
Headache & $156(17.7 \%)$ & $17,45(13.1 \%)$ & $1.4(1.2-1.7)$ & $<0.001$ \\
Injury mechanism & $490(55.7 \%)$ & $3784(28.3 \%)$ & $3.2(2.8-3.6)$ & $<0.001$ \\
Intoxication & $149(16.9 \%)$ & $718(5.4 \%)$ & $3.6(3.0-4.3)$ & $<0.001$ \\
Prev. NCH & $37(4.2 \%)$ & $186(1.4 \%)$ & $3.1(2.2-4.5)$ & $<0.001$ \\
Antiplatelet therapy & $180(20.5 \%)$ & $1,186(8.9 \%)$ & $2.6(2.2-3.1)$ & $<0.001$ \\
\hline
\end{tabular}

^ Intracranial lesions were: intracerebral haematoma/brain contusion(s), 591 (67.1\%) subarachnoid haemorrhage, 193 (21.9\%) subdural haematoma, 329 (37.4\%) epidural haematoma, 63 (7.2\%). GCS: Glasgow coma scale, LOC: loss of consciousness, Prev. NCH: history of neurosurgical intervention.

* Only in patients who experienced loss of consciousness or amnesia. 


\section{Table 3}

Multivariable model for prediction of intracranial lesions in subjects after mild head injury.

\begin{tabular}{|c|c|c|c|c|c|c|}
\hline \multirow[b]{2}{*}{ Variables } & \multicolumn{2}{|c|}{ Main Variables } & \multicolumn{2}{|c|}{ Antiplatelet Drugs } & \multicolumn{2}{|c|}{ Antiplatelet Drugs, Age $\geq 75$ years } \\
\hline & OR $(95 \% \mathrm{CI})$ & $P$ value & OR $(95 \% \mathrm{CI})$ & $P$ value & OR $(95 \% \mathrm{CI})$ & $P$ value \\
\hline GCS 14 & $17.8(13.3-23.9)$ & $<0.001$ & $17.8(13.2-23.8)$ & $<0.001$ & $17.9(13.3-24.0)$ & $<0.001$ \\
\hline Amnesia & $0.8(0.7-1.1)$ & 0.153 & $0.8(0.7-1.0)$ & 0.101 & $0.8(0.7-1.1)$ & 0.163 \\
\hline $\begin{array}{l}\text { Suspected skull } \\
\text { fracture }\end{array}$ & $13.9(8.9-21.7)$ & $<0.001$ & $13.8(8.9-21.6)$ & $<0.001$ & $13.9(8.9-21.8)$ & $<0.001$ \\
\hline Vomiting & $17.1(12.0-24.4)$ & $<0.001$ & $17.4(12.1-25.0)$ & $<0.001$ & $17.9(12.4-25.7)$ & $<0.001$ \\
\hline Age $\geq 65$ yrs & $1.7(1.3-2.2)$ & $<0.001$ & $1.2(0.9-1.7)$ & 0.202 & -- & -- \\
\hline Age $\geq 75$ yrs & -- & -- & -- & -- & $1.4(1.0-1.9)$ & 0.037 \\
\hline Coagulopathy & $5.583 .7-8.0$ & $<0.001$ & $7.4(5.0-11.1)$ & $<0.001$ & $7.1(4.8-10.6)$ & $<0.001$ \\
\hline Neurological deficit & $115.7(76.9-174.1)$ & $<0.001$ & $115.6(76.6-174.5)$ & $<0.001$ & $117.1(77.5-176.8)$ & $<0.001$ \\
\hline Seizure & $3.4(2.1-5.6)$ & $<0.001$ & $3.4(2.0-5.5)$ & $<0.001$ & $3.4(2.0-5.5)$ & $<0.001$ \\
\hline LOC & $3.0(2.3-3.9)$ & $<0.001$ & $3.1(2.4-4.0)$ & $<0.001$ & $3.2(2.5-4.1)$ & $<0.001$ \\
\hline Visible trauma & $0.9(0.7-1.2)$ & 0.888 & $0.9(0.7-1.1)$ & 0.886 & $0.9(0.7-1.2)$ & 0.526 \\
\hline Headache & $1.1(0.8-1.4)$ & 0.730 & $1.0(0.8-1.4)$ & 0.775 & $1.0(0.8-1.4)$ & 0.763 \\
\hline Injury mechanism & $1.2(0.9-1.5)$ & 0.244 & $1.2(0.9-1.5)$ & 0.253 & $1.1(0.8-1.4)$ & 0.517 \\
\hline Intoxication & $1.0(0.7-1.5)$ & 0.939 & $1.0(0.7-1.5)$ & 0.943 & $1.0(0.7-1.5)$ & 0.972 \\
\hline Previous neurosurgery & $0.5(0.2-1.2)$ & 0.116 & $0.5(0.2-1.2)$ & 0.139 & $0.5(0.2-1.2)$ & 0.140 \\
\hline Antiplatelet drugs & -- & -- & $2.8(2.0-3.9)$ & $<0.001$ & $2.7(1.9-3.7)$ & $<0.001$ \\
\hline
\end{tabular}




\section{Table 4}

Sensitivity, specificity, operating characteristics curve and negative predictive value of the items selected by different variable combinations in the prediction of intracranial lesions in subjects with mild head injury.

\begin{tabular}{|c|c|c|c|c|c|c|}
\hline & \multicolumn{2}{|c|}{ Main Variables } & \multicolumn{2}{|c|}{ Antiplatelet Drugs } & \multicolumn{2}{|c|}{ Antiplatelet Drugs, Age $\geq 75$ yrs } \\
\hline & Yes & No & Yes & No & Yes & No \\
\hline \multicolumn{7}{|l|}{ Intracranial Lesions } \\
\hline Variables present & 866 & 7,869 & 860 & 4,831 & 877 & 7,207 \\
\hline Variables absent & 14 & 5,479 & 20 & 8,517 & 3 & 6,141 \\
\hline Sensitivity (\%) & \multicolumn{2}{|c|}{$98.4(95 \%$ CI 97.3 - 99.0) } & \multicolumn{2}{|c|}{97.7 (95\%CI 96.4 - 98.5) } & \multicolumn{2}{|c|}{99.7 (95\%CI 98.9 - 99.8) } \\
\hline Specificity (\%) & \multicolumn{2}{|c|}{$41.1(95 \%$ CI $40.2-41.9)$} & \multicolumn{2}{|c|}{$63.8(95 \%$ CI $63.0-64.6)$} & \multicolumn{2}{|c|}{$54.0(95 \%$ CI $53.1-54.8)$} \\
\hline AUC & \multicolumn{2}{|c|}{0.697 (95\%CI $0.690-0.705)$} & \multicolumn{2}{|c|}{$0.807(95 \%$ CI $0.803-0.814)$} & \multicolumn{2}{|c|}{$0.783(95 \%$ CI $0.761-0.775)$} \\
\hline NPV\% & \multicolumn{2}{|c|}{99.7} & \multicolumn{2}{|c|}{99.8} & \multicolumn{2}{|c|}{100} \\
\hline TC Ordering Rate $\%$ & \multicolumn{2}{|c|}{61.4} & \multicolumn{2}{|c|}{40.0} & \multicolumn{2}{|c|}{49.3} \\
\hline
\end{tabular}

${ }^{\#}$ Fisher's exact test, $\mathrm{P}<0.001$. CI denotes confidence intervals, AUC: Area Under the Curve. NPV: negative predictive 


\section{Appendix}

\section{Table 1}

Relative significance of age and antiplatelet drug use in the prediction of intracranial lesions after mild head injury in different models considering age either as continuous variable or as decades (ordinal) or as categorical with different cut-offs. The combination and odds ratio of classical variables identified by the main decision instruments (GCS score, neurological deficit, loss of consciousness, suspected skull fracture, vomiting, seizures, coagulopathy) are not reported in detail.

\begin{tabular}{lcc}
\hline Variable & Age & Antiplatelet Agents \\
& OR $(\mathbf{9 5 \%} \mathbf{C I})$ & OR $(\mathbf{9 5} \% \mathbf{C I})$ \\
Age (continuous) & $1.01(1.00-1.02)$ & $2.29(1.63-3.21)$ \\
Age (decades) & $1.12(1.05-1.19)$ & $2.30(1.64-3.22)$ \\
Age Cut-off (nominal) & & \\
$\geq 50 \mathrm{yrs}$ & $1.32(0.98-1.78)$ & $2.69(1.94-3.75)$ \\
$\geq 55 \mathrm{yrs}$ & $1.33(0.99-1.79)$ & $2.66(1.91-3.72)$ \\
$\geq 60 \mathrm{yrs}$ & $1.26(0.94-1.70)$ & $2.72(1.94-3.82)$ \\
$\geq 65 \mathrm{yrs}$ & $1.28(0.96-1.74)$ & $2.68(1.91-3.70)$ \\
$\geq 70 \mathrm{yrs}$ & $1.24(0.92-1.67)$ & $2.77(1.99-3.87)$ \\
$\geq 75 \mathrm{yrs}$ & $1.38(1.02-1.86)$ & $2.65(1.91-3.70)$ \\
$\geq 80 \mathrm{yrs}$ & $1.97(1.48-2.63)$ & $2.38(1.72-3.28)$ \\
$\geq 85 \mathrm{yrs}$ & $2.88(2.09-3.96)$ & $2.27(1.65-3.12)$ \\
$\geq 90 \mathrm{yrs}$ & $4.41(2.87-6.77)$ & $2.25(1.63-3.10)$ \\
\hline
\end{tabular}

